\title{
Arrayed Primer Extension in the "Array of Arrays" Format: A Rational Approach for Microarray-Based SNP Genotyping
}

\author{
NIELS G.F. KLIT $\varnothing,{ }^{1}$ QIHUA TAN,${ }^{1}$ METTE NYEGAARD, ${ }^{2}$ KLAUS BRUSGAARD, ${ }^{1}$ \\ MADS THOMASSEN,${ }^{1}$ CHARLOTTE SKOUBOE,${ }^{1}$ JESPER DAHLGAARD,${ }^{3}$ and TORBEN A. KRUSE ${ }^{1}$
}

\begin{abstract}
This study provides a new version of the arrayed primer extension (APEX) protocol adapted to the 'array of arrays' platform using an instrumental setup for microarray processing not previously described. The primary aim of the study is to implement a system for rational cost-efficient genotyping where multiple singlenucleotide polymorphisms (SNPs) and individuals are genotyped on each microarray slide. Genotyping results are collected across 185 healthy Danish subjects and 76 SNPs on chromosome 3q13.31, because linkage to atopic disease phenotypes have been suggested in the Danish population. Linkage disequilibrium (LD) results from the experimental data are used in a novel comparison to baseline data defined by the international HapMap SNP database. Comparison on the LD results reveals a strong linear correlation irrespective of LD measure considered: $R^{2}\left(\mathrm{D}^{\prime}\right)=0.73$ and $R^{2}\left(r^{2}\right)=0.54$. In conclusion, our results show that this setup is strong enough to support high-throughput genotyping, and these observations support that the HapMap genotype resource is important for defining SNP panels aimed at gene mapping in local subpopulations from Europe.
\end{abstract}

\section{INTRODUCTION}

M ICROARRAYS CONSISTING of multiple identical subarrays have been used to explore different strategies of singlenucleotide polymorphism (SNP) genotyping including, for instance, allele-specific primer extension and cyclic mini sequencing (Pastinen et al., 2000; Syvanen, 2001; Lovmar et al., 2003). The 'array of arrays' format used in these studies (Fig. 1A) is presented as a potential high-throughput genotype platform, because up to 80 individuals can be examined on each microarray slide. In other words, only a limited number of the addressed microarrays will be needed to collect data across hundreds of individuals if a robust protocol is available. Arrayed primer extension (APEX) provides a potential example of such a protocol (Kurg et al., 2000; Tonisson et al., 2000; Tebbutt et al., 2004), but has not previously been adapted to the 'array of arrays' format.

A difference between minisequencing and APEX is that typical minisequencing relies on a two-step procedure, whereas APEX relies on a single-step procedure (Fig. 2). Minisequenc- ing is single-base extension (SBE) in a liquid phase carried out in a separate reaction tube (step 1) using tag-labeled oligonucleotides (Syvanen, 2001). Anti-tag sequences, spotted on microarray glass slides, are then used to sort all the SBE products that contain the genotypic information (step 2). In contrast, APEX is, in principle, simpler, because instantaneous SBE of all immobilized SNP flanking primers is achieved when the fragmented PCR amplicons are exposed directly to the microarray (Tonisson et al., 2000). The parallel SNP detection capacity of APEX appears to be high, and this provides a potential cost-friendly genotyping protocol with low reagents consumption per SNP. For instance, the standard 'single subject per microarray approach' has been used to explore linkage disequilibrium (LD) across chromosome 22 with more than 900 SNPs examined (Dawson et al., 2002) and for genetic association studies (Landi et al., 2005; Maron et al., 2005) as well.

Here, we combine the 'array of arrays' platform with a new modified version of the APEX protocol to ease genotyping across hundreds of individuals using only a few microarray slides. The method is adapted to a low-cost instrumentation

\footnotetext{
${ }^{1}$ Department of Biochemistry, Pharmacology and Genetics, Human MicrroArray Centre, Odense University Hospital, Odense, DK-5000 Odense C, Denmark.

${ }^{2}$ Institute of Human Genetics, University of Aarhus, Denmark.

${ }^{3}$ Department of Haematology, Aarhus University Hospital, Aalborg, Denmark.
} 
A

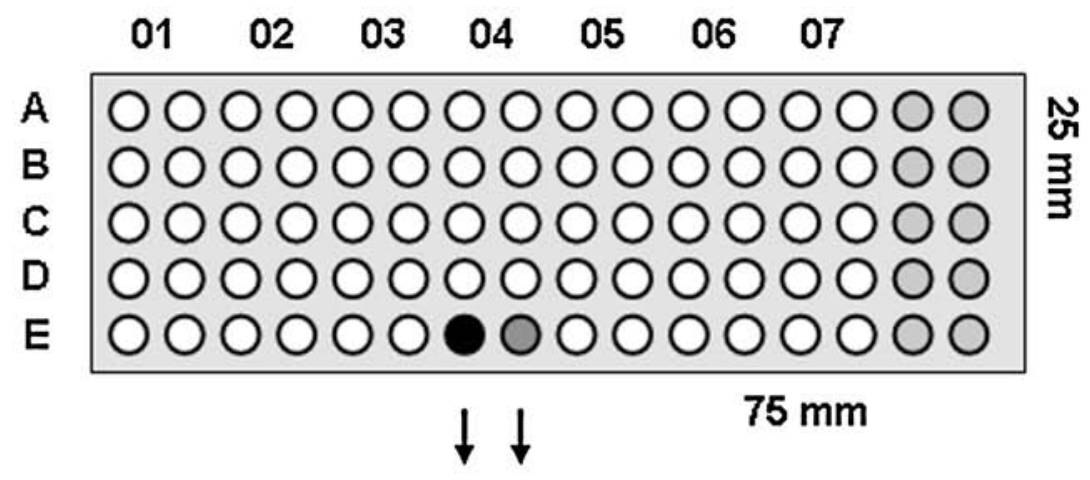

Individual E04
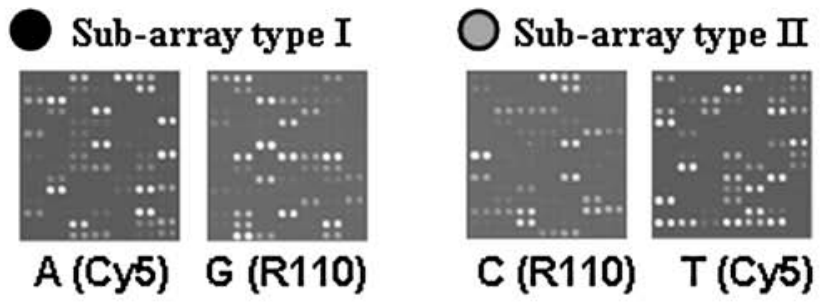

\section{Nucleotide specific scan images}

B

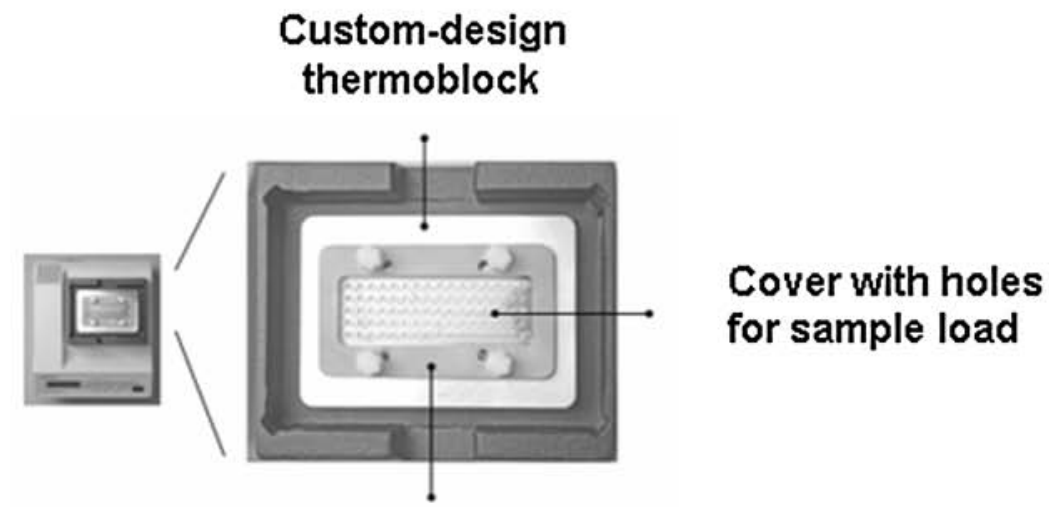

\section{Pressure adjustment ring}

FIG. 1. A: Schematic illustration of the "array of arrays" format described in the text. The locations of the different subarrays on a microarray slide are indicated as circles; two subarrays are needed to genotype each subject. Subarray type I denotes the location of an array specific for nucleotides A and G. Subarray type II denotes location of an array specific for nucleotides C and T. An example of tiff-file export, generated by a microarray scanner, is shown below the schematic drawing. B: Equipment used for microarray slide processing. An aluminium ring with a depression and four screws is used to control the pressure between the polycarbonate cover and the rack bottom where the microarray slide and the silicone rubber grid are inserted.

setup for microarray processing not previously described (Fig. 1B). Our approach is adapted to the 96-well plate format to support various high-throughput genotyping application of specific importance to our hospital facility. A panel of 96 potential SNP loci is chosen across the chromosome $3 q 13.31$ region and used to test the system. Genotype data from the 3q13.31 SNPs are collected and used in LD analysis where experimental results are compared to HapMap data.

\section{MATERIALS AND METHODS}

\section{Experimental design}

The SNPs in our study was defined by choosing 96 potential SNP loci on the National Center for Biotechnology Information (NCBI) SNP database (http://www.ncbi.nlm.nhi.gov) between two microsatellite DNA markers D3S1278 and 


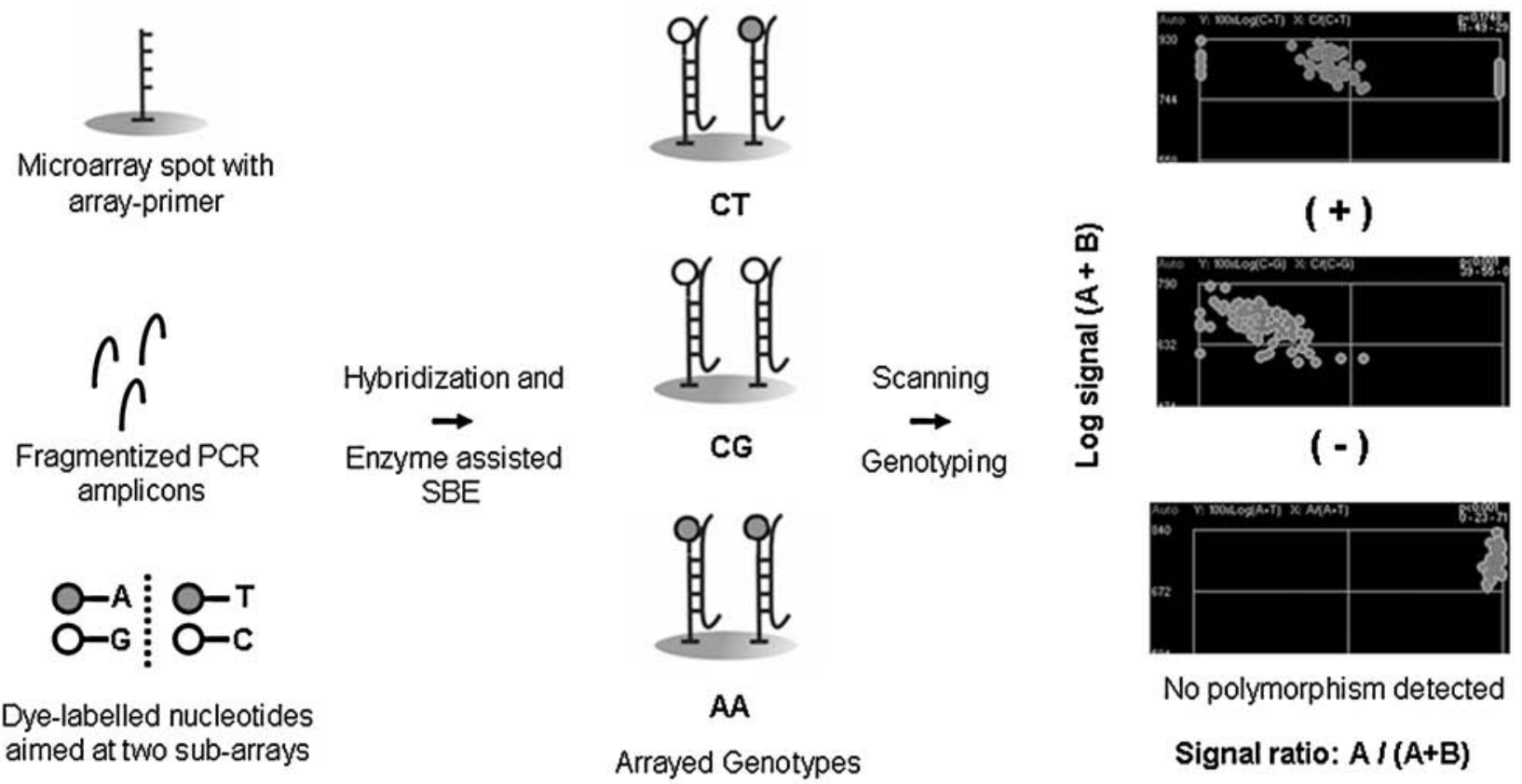

FIG. 2. Summary of the molecular reaction principle and work flow of APEX-based SNP genotyping with essential reagents depicted to the left. All array primers are extended with dideoxynucleotides during an enzyme-assisted single-step procedure (SBE). The PCR amplicons are allowed to hybridize with their corresponding SNP primer sequences on the microarray, with all SNPs detected simultaneously. Different genotype cluster representations of the potential SNP loci examined are depicted to the right, and A and B refer to spot intensities of the detected SNP alleles. Only informative markers (+) are included in the LD application. Note, genotype results may originate from two subarrays (CG genotype shown) in the described two-dye APEX format.

D3S1303 on chromosome 3q13.31. The physical distance between these two microsatellites is approximately $2.84 \mathrm{Mb}$ and defines the borders of a linkage signal that we expect to harbor a gene for atopic diseases (Brasch-Andersen et al., 2006). SNPs in that region were randomly chosen for genetic fine mapping (LD relation not considered) with a preference for reported heterozygosity and location in gene loci. One array primer and a PCR primer pair were chosen per SNP locus and ordered from the Illumina Inc. synthesis service. The ChipDesigner ${ }^{\mathrm{TM}}$ Software (BioData, Estonia) was used for parallel design of all oligonucleotides in the SNP panel. Oligonucleotide sequences are available as part of the supplementary section (Supplementary Tables 1-3, accessible at http://www1.sdu.dk/Videncentret/ arkiv/SupplementaryTables.pdf). The design of our experiments diverge from the classic APEX design in which both SNP flanking primers are used to determine genotypes (Tebbutt et al., 2004). We test only one array primer per SNP. The rationale for this approach was to gain additional genotype information from other SNPs rather than confirming already known information due to the sense/antisense-based APEX genotyping.

The majority of SNPs considered in the present study are located in two validated genes and one major hypothetical gene locus: growth-associated protein (GAP43), limbic-system-associated membrane protein (LSAMP), and gene locus LOC389142, respectively (Supplementary Table 4, accessible at http:// www1.sdu.dk/Videncentret/arkiv/SupplementaryTables.pdf). Detailed data quality comparison between our single primer approach and the commonly applied bidirectional APEX genotyping approach has to our knowledge not been performed. A series of pilot experiments prior to the reported experiments showed a potential increase in the number of usable SNPs if the bidirectional approach was used, as at least one of the array primers may provide high-quality genotypes. However, in terms of expense of microarray primers, these experiments showed that for most SNPs we would end up paying twice for the same genotypes. This is avoided with our unidirectional approach and explains why we prefer this approach in the present study. Result discrepancies between primers of the same SNP were not found if both primers showed high-quality clustering (Klitø, 2006).

\section{Purified and fragmented PCR amplicons}

Twenty nanograms of DNA ( $20 \mathrm{ng} / \mu \mathrm{l})$ was used as template for all PCR reactions (conditions available for the 29 PCR reactions upon request). DNA from 185 unrelated healthy Danes were included and organized in two DNA sample boxes in the 96-well plate format. PCR was performed in the 96-well plate format as well following the procedure specified in other APEX reports (Kurg et al., 2000; Tonisson et al., 2000). Multiplex PCR groupings of primer pairs were specified by the BioData software, but further subdivided if PCR quality was observed low on gels.

The products were collected for each of the 185 subjects using Microcon ${ }^{\circledR}$-PCR filters plates (Millipore), a convenient setup for high-throughput applications in the 96-well plate format. Ten microliters from each PCR reaction were added to create subject-specific PCR pools. Each pool was cleaned with 100 $\mu \mathrm{l}$ of milliQ- $\mathrm{H}_{2} \mathrm{O}$ after initial filtration in the filtration plate. 
The cleaned PCR products were redissolved and transferred to new set of tubes in $50 \mu \mathrm{l}$ of milliQ- $\mathrm{H}_{2} \mathrm{O}$ and dried to pellet on a Speedy-Vac centrifuge. The PCR amplicons were then redissolved in $16 \mu \mathrm{l}$ of milliQ- $\mathrm{H}_{2} \mathrm{O}$ and fragmented by SAP/UNG treated with uracil- $N$-glycosylase/shrimp alkaline phosphatase (Fermantas) following standard procedures $\left(1 \mathrm{~h}\right.$ at $37^{\circ} \mathrm{C}$ followed by $10 \mathrm{~min}$ of heat inactivation of enzymes at $95^{\circ} \mathrm{C}$ ). We added $1 \mu \mathrm{l}$ of each enzyme stock plus $2 \mu \mathrm{l}$ of UNG reaction buffer per PCR product pool. The product pools were then diluted further by addition of $20 \mu \mathrm{l}$ of milliQ- $\mathrm{H}_{2} \mathrm{O}$. The fragmented and diluted PCR products were organized in subjectlabeled strips matched in the format of the microarrays and stored at $4^{\circ} \mathrm{C}$.

\section{Microarray fabrication and processing}

Code-link ${ }^{\mathrm{TM}}$-activated microarray slides (AmershamBiosciences) were printed with 5' amine-modified extension array primers using the recommended protocols supplied by the company. A VersArrayer ChipWriterPro microarray spotter (BioRad), equipped with SMP2.5 quill pins (TeleChem International Inc.), was used to spot 70 identical subarrays consisting of the primer-specific spots of the SNPs (spot center-to-center spacing was $175 \mu \mathrm{m}$, duplicated spots of all array primers). This microarray format enables genotyping of 35 individuals on each microarray slide (Fig. 1A). The rack parts needed to process these microarrays were designed in cooperation with a local company FIMEK (http://www.fimek.dk). Silicone rubber grids were prepared as previously described (Pastinen et al., 2000) to define the independent reaction chambers on each microarray slide. The bottom of each reaction chamber contains one of the 70 identical subarrays.

The APEX protocol (Kurg et al., 2000) was adjusted to support our two-dye setup. All mixing of reagents and PCR products must be carried out on ice to assure experiments of high quality. We use only two dyes because our scanner configuration does not support four dyes. However, we emphasize that the four-dyes approach is preferable, because the sample throughput will double and the setup complexity will be reduced as only one subarray is needed per subject. The reaction mixture for genotyping an individual was composed as follows: $3.4 \mu \mathrm{l}$ of diluted and denatured PCR pool $\left(95^{\circ} \mathrm{C}, 15 \mathrm{~min}\right)$ was mixed with $5 \mu$ l of master mix containing 0.5 units of Thermo Sequenase (AmershamBiosciences), $1.5 \times$ reaction buffer, and $0.5 \mu M$ of each dideoxynucleotide (PerkinElmer) aimed at the two subarray types needed to detect the four DNA bases in our two-dye APEX system. Each master mix should ideally support three 'array of arrays' experiments to limit experimental variation across slides-important if slides are genotyped together. The nucleotides of master mixes 1 and 2 were Cyanine5ddATP, ddCTP, R110-ddGTP, ddTTP (aimed at 'Sub-array type I', Fig. 1A) and ddATP, R110-ddCTP, ddGTP, Cyanine5ddTTP (aimed at 'Sub-array type II', Fig. 1A), respectively. In the present experiments, we use R110-labeled nucleotides instead of the more typically used Cyanine3-labeled nucleotides (Fortina et al., 2000), because pilot experiments (results not shown) involving various nucleotide combinations showed that the R110-labeled nucleotides performed well together with the Cyanine5-labeled nucleotides.

A total of $7.5 \mu \mathrm{l}$ of the final reaction mixtures, containing all the necessary reagents, were added to separate subarray chambers on the microarray by use of an eight-channel pipette. A short spin-down step $(15 \mathrm{sec})$ of the preheated slide, mounted in the slide rack, was performed to ensure exposure of all 70 subarrays to the reaction mixture. A ThermoStat Plus heater (Fig. 1B), equipped with a MTP box (Eppendorf), was used to supply heat $\left(58^{\circ} \mathrm{C}, 20 \mathrm{~min}\right)$. Slides were then unmounted quickly, placed in a slide holder, and transferred to a glass staining jar (MerckEurolab) with magnetic bar agitation. The slides were washed at room temperature: $3 \mathrm{~min}$ in $0.2 \times \mathrm{SSC}, 0.1 \%$ sodium dodecyl sulfate (SDS), and $2 \times 3 \mathrm{~min}$ in distilled water. Compressed particle-free air was used to dry the slides prior to scanning. Scanning was carried out immediately following drying because Cyanine5 is known to degrade fast if exposed to atmospheric ozone. Scanning and genotyping were performed as described elsewhere (Kurg et al., 2000; Tonisson et al., 2000; Tebbutt et al., 2004). An arrayWoRx ${ }^{\circledR}$ chip reader (AppliedPrecision) was used to obtain nucleotide-specific images (tiff-file format) needed for genotyping by the Genorama $^{\text {TM }}$ software package (AsperBiotech). Base calls were finally listed in columns as single nucleotide letters A, C, G, and $\mathrm{T}$ (MS Excel) and reformatted to support statistical analysis in the linkage-style data format.

\section{LD analysis}

Chromosome 3q13.31 genotype data were sorted according to physical map location of the SNPs across the chromosome region. The program EMLD (available at http://epi.mdanderson.org) was used to estimate LD across marker pairs using an expectation maximization algorithm (Excoffier and Slatkin, 1995). Results of two popular LD measures were reported by the program, i.e., Lewontin's $D^{\prime}$ (Lewontin, 1964) and Hill and Robertsons' $r^{2}$ (Hill and Robertson, 1968). LD is simply defined as a nonrandom association of alleles between linked loci. Readers interested in more detailed information concerning the mathematical definitions of $D^{\prime}$ and $r^{2}$ should refer to Jorde (2000) or Weiss and Clark (2002).

The LD results were portrayed in two separate LD maps by the program Gold (Abecasis and Cookson, 2000). These maps were merged to form Fig. 3 and are shown in gray-scale format. Essentially, an experimental LD block was defined as present if both plots support a grey-to-white LD color coding along the diagonal of the figure ( $L D \geq 0.5$ ). In contrast, $L D$ was defined as absent across neighboring blocks if the color coding tends to be blue (LD approaching 0.0 but always $<0.4$ ). Specific marker pairs confirming LD results, within and across blocks as either present $(\mathrm{LD}+)$ or absent $(\mathrm{LD}-)$, were chosen if consistent results for both LD measures $\left(D^{\prime}\right.$ and $\left.r^{2}\right)$ were observed. This approach was used to assure reliable LD results among the specific marker pairs and to provide the data points for comparison of experimental results relative to HapMap. The required genotype data from HapMap was downloaded from the Web site http://www.hapmap.org assuming a Caucasian (CAU) population sample. Linear regression was employed to estimate the statistical correlation $\left(R^{2}\right)$ between the EMLD-generated LD data representing the two population samples of European origin. To assist comparison, a close neighboring SNP, within the same haplotype block, was chosen from HapMap if a SNP in our data was not found in the database. 


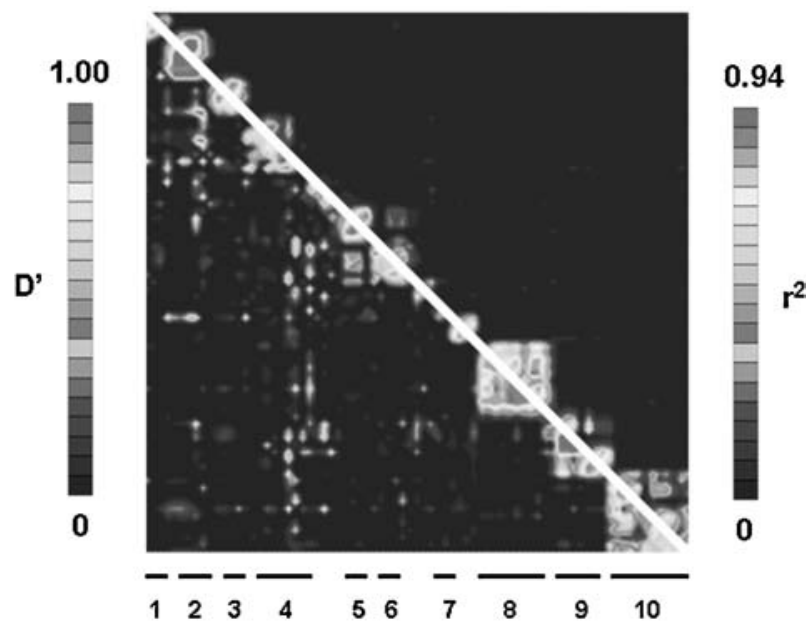

FIG. 3. Gold plots showing the distribution of LD between the SNPs dispersed along the studied chromosome 3q13.31 region, $D^{\prime}$ below diagonal and $r^{2}$ above diagonal, respectively. Color-coded LD scaling, including the detected minimum and maximum of both LD measures, is indicated at each side of the plots. LD blocks identified through visual inspection of the plots are underlined and assigned a number from 1 to 10 .

\section{RESULTS}

Among the 96 SNPs tested on chromosome 3, data were extracted from 76 SNPs. These markers were informative and of high quality, as depicted in Fig 2. Five of the SNPs showed no heterozygosity $\left(\mathrm{H}_{\mathrm{o}}\right)$ in the NCBI SNP database. The following heterozygosities were observed: $\operatorname{rs} 1381800\left(\mathrm{AG}, \mathrm{H}_{\mathrm{o}}=0.48\right)$, rs938113 (CG, $\left.\mathrm{H}_{\mathrm{o}}=0.30\right)$, rs939032 (AG, $\left.\mathrm{H}_{\mathrm{o}}=0.51\right)$, rs4378954 (CT, $\left.H_{o}=0.14\right)$, and $\operatorname{rs} 1499978\left(A G, H_{o}=0.28\right)$. The most frequently observed allele and its type (A, C, G, or
T) are reported for all informative SNPs in the supplementary material.

An average call rate of $97.2 \%$ was found for 67 out of the 76 SNPs, and a reduced call rate (around 50\%) was found for the remaining 9 SNPs. This was explained by our genotyping approach, where three microarray experiments (DNA sample box one or two) were genotyped together. If genotype clustering was low from one DNA sample box, Fig. $2(-)$, then we deleted these calls to avoid introducing potential genotyping errors.

In addition to the 76 SNPs from which data are included, 20 of the potential SNP loci examined were left out of the LD application due to the following reasons: (1) eight were apparently not polymorphic, (2) five were only rarely polymorphicaround $1 \%$ heterozygotes were found; and (3) the remaining SNPs tested were too difficult to type due to low-quality clustering across all experiments, typically associated with faint signal intensities.

The LD results of the chromosome 3q13.31 data are summarized below. Considerably more variation in LD was observed for $D^{\prime}$ than $r^{2}$ resulting in a more complex LD pattern (Fig. 3). A visual inspection of the two LD maps shows presence of 10 LD blocks confirmed by $D^{\prime}$ and $r^{2}$. LD blocks $1-6$ span the genes LSAMP and GAP43 (SNP1-35). In contrast, group 7-10 does not cover any known genes, but one major hypothetical gene locus, LOC389142 (blocks 9 and 10, SNP61-76). Linear regression analysis of the LD values involving both the experimental data and data from HapMap is reported in Table 1 . The following correlations were found: $R^{2}$ $\left(D^{\prime}\right)=0.73$ and $R^{2}\left(r^{2}\right)=0.54$.

\section{DISCUSSION}

\section{Advantages and perspectives of the genotyping system}

Simple, robust, and cost-friendly protocols are needed to exploit the genotyping potential of the spotted microarray format.

Table 1. Chosen LD Results Representing Experimental Data and HapMap Data

\begin{tabular}{|c|c|c|c|c|}
\hline Block & $L D+$ & $L D-$ & $L D+\left(\mathrm{D}^{\prime}\right) L D-$ & $L D+\left(\mathrm{r}^{2}\right) L D-$ \\
\hline $01(1-3)$ & $2-3$ & $3-5$ & $0.97,0.97 / 0.19,0.29$ & $0.59,0.64 / 0.01,0.01$ \\
\hline 02 (4-9) & $5-7$ & $7-11 *$ & $0.99,0.83 / 0.25,0.42$ & 0.89, 0.24/0.01, 0.05 \\
\hline $03(10-14)$ & $11 *-13$ & $13-16$ & $0.93,1.00 / 0.09,0.02$ & $0.82,0.95 / 0.01,0.01$ \\
\hline $04(16-22)$ & $18-20$ & $20-23$ & $0.96,0.94 / 0.10,0.44$ & $0.49,0.33 / 0.01,0.09$ \\
\hline $05(28-31)$ & $29-31 *$ & $28-32$ & $0.94,1.00 / 0.34,0.06$ & $0.82,1.00 / 0.05,0.01$ \\
\hline $06(33-38)$ & $35-36$ & $33-32$ & $0.81,0.61 / 0.28,0.63$ & $\mathbf{0 . 6 1}, \mathbf{0 . 1 1} / 0.01,0.05$ \\
\hline $07(44-46)$ & $45 *-46$ & $46-47$ & $0.86,0.99 / 0.02,0.37$ & $0.47,0.39 / 0.01,0.09$ \\
\hline $08(48-56)$ & $48-50$ & $50-58 *$ & $0.92,0.95 / 0.06,0.04$ & $0.71,0.87 / 0.00,0.00$ \\
\hline $09(59-65)$ & $59 *-62 *$ & $65-67$ & $0.95,0.51 / 0.11,0.42$ & $0.77,0.01 / 0.01,0.10$ \\
\hline $10(66-76)$ & $68-69$ & $68-65$ & $0.92,0.99 / 0.02,0.08$ & $0.81,0.94 / 0.00,0.00$ \\
\hline
\end{tabular}

LD blocks 1-10 and SNPs within blocks (shown in brackets) are reported in the first column. The SNP identification codes (SNP 1-76) are shown in separate columns for each comparison specified in the LD + and LD - columns. LD results for $D^{\prime}$ and $r^{2}$ are reported in the last two columns. The left- and right-hand sides of the slash (/) specify the two LD values compared. The first value denotes the experimental result and the second value in italics denotes the corresponding HapMap result. SNPs supplied with an asterisk (*) indicate direct comparison was not possible, because these SNPs were not part of HapMap. Subsequently, SNP11, 31, 45, 58, 59, and 62 were replaced by HapMap markers rs283392, rs9835427, rs4132417, rs 1501878, rs1610148, and rs10934434 to assist comparison. 
This study has introduced a new two-dye version of the APEX protocol combined with an 'array of arrays' platform. The setup is designed specifically for our hospital facility using a costfriendly instrument for microarray processing not previously described (the ThermoStat Plus, Eppendorf). One specific advantage of this instrument equipped with our special designed 'array of arrays' rack (Fig. 1B) is that, as the heating device is already involved in various temperature-regulated applications in our molecular genetic laboratory, the system is, therefore, a cost-effective exploration of an equipment already in frequent use.

Importantly, the setup is cheap to implement if other essential microarray hardware are available (spotter and scanner) and provides a relatively high multiplex capacity compared to other SNP detection systems, for example, the very flexible and popular sequencing automaters. Our system provides the same assay flexibility but with increased multiplex capacity, i.e., in principle, complete control of SNP choice within subarrays containing up to approximately 96 SNPs in the reported microarray format. At the same time, our system supports a more costefficient exploration of reagents compared to sequencing automates as only $7.5 \mu \mathrm{l}$ of the reaction mixture is necessary per DNA sample (each subarray) for simultaneous detection of all SNPs in our panel. Other chip-based SNP genotyping platforms (Affymetrix, Genehip ${ }^{\circledR}$ or Illumninas BeadArray ${ }^{\mathrm{TM}}$ technology) support gene mapping, even on the genome-wide level (Syvanen, 2005), but the SNP panels used are defined by the companies and this may not always be desirable

We believe the focused 'array of arrays' approach will remain attractive, particularly along with the gradual decoding of genome through genome-wide mapping. As the number of potential marker panels needed to study specific gene-phenotype relations in clinical-related research (risk assessment, pharmacogenomics, or gene mapping) will increase in the future, it may be useful to consider only the most important SNPs that have been found to influence a given trait or disease and avoid genotyping SNPs in the genome known not to be relevant. Moreover, the 'array of arrays' platform also provides a convenient setup for innovative exploration of new SNP detection strategies or modifications to already described methods.

\section{Comparison of experimental result and HapMap results}

High-quality genotype data of the international HapMap database is freely available for designing high-resolution gene mapping projects (Hinds et al., 2005; The International HapMap Consortium 2005; Thorisson et al., 2005). These data are also an obvious target for exploitative comparison of chromosomal focused LD results as described below. In fact, involvement of HapMap data in various application types is encouraged by the HapMap organization to gain a deeper understanding of, for instance, human genome evolution. Previous studies have documented reliable genotyping through comparison of APEX-generated data with data from other SNP detection platforms (Jaakson et al., 2003; Kaminski et al., 2005; Schrijver et al., 2005). Here we address experimental data quality through a novel indirect comparison of our LD results to baseline data from HapMap because previous examinations showed that the European (in this report 185 healthy Danes) and in particular the Caucasians population sample from HapMap are comparable (Weiss and Clark, 2002). The HapMap data involved represents 90 Caucasian subjects (30 family trios).

A genetic relationship across the two population samples is confirmed through our LD analysis. Strong linear correlation is detected both for $D^{\prime}\left(R^{2}=0.73\right)$ and $r^{2}\left(R^{2}=0.54\right)$. That is, both LD measures support a clear correlation between the experimental results and the HapMap data confirming evolutionary stable LD patterns. Such patterns are important in terms of the gene mapping study design (Gabriel et al., 2002). However, there are three comparisons that negatively influence the regression for $r^{2}$, shown as bold numbers in Table 1; thus, LD is confirmed across our experimental results by $D^{\prime}$ as well as $r^{2}$. In one case, this may be explained through our SNP replacement procedure (block 9, $r^{2} \mathrm{LD}+$, Table 1 ) because, occasionally, genotyped markers were substituted by nearby validated HapMap SNPs to assist comparison. The remaining pair of comparisons (block $2 \& 6, r^{2} \mathrm{LD}+$, Table 1) serves as examples of differences across experimental results and HapMap results, although all individuals originate from Europe. Nevertheless, such potential differences may not negatively influence gene mapping if they remain a rare phenomenon, as suggested through our examination.

In summary, this report provides a new version of the APEX protocol adapted to the 'array of arrays' format using a costfriendly setup for microarray slide processing not previously described. Our novel LD application, where chosen HapMap data is used as baseline relative to our experimental data, indicates that the system is strong enough to support focused LD mapping projects. The limitation regarding the number of SNPs of the described system is determined primarily by the spotspacing within subarrays. We are confident that APEX in the 'array of arrays' format provides a very simple, attractive, yet highly flexible setup for a wide-spectrum of high-throughput genotyping projects involving hundreds of subjects and any focused SNP panel of interest through a cost-effective exploration of reagents.

\section{ACKNOWLEDGMENTS}

We thank the Danish Biotechnology Instrument Centre (DABIC) and the following oligonucleotide providers Unest, DNA Technology, Taq Copenhagen, Eurogentech, and Medprobe for supplying oligonucleotides to the pilot experiment addressed in the manuscript.

\section{REFERENCES}

Abecasis GR, Cookson WO (2000) GOLD—graphical overview of linkage disequilibrium. Bioinformatics 16:182-183.

Brasch-Andersen C, Haagerup A, Borglum AD, Vestbo J, Kruse TA (2006) Highly significant linkage to chromosome 3 q13.31 for rhinitis and related allergic diseases. J Med Genet. 43:e10.

Dawson E, Abecasis GR, Bumpstead S, Chen Y, Hunt S, Beare DM, Pabial J, Dibling T, Tinsley E, Kirby S, Carter D, Papaspyridonos M, Livingstone S, Ganske R, Lohmussaar E, Zernant J, Tonisson N, Remm M, Magi R, Puurand T, Vilo J, Kurg A, Rice K, Deloukas P, Mott R, Metspalu A, Bentley DR, Cardon LR, Dunham I (2002) A 
first-generation linkage disequilibrium map of human chromosome 22. Nature 418:544-548.

Excoffier L, Slatkin M (1995) Maximum-likelihood estimation of molecular haplotype frequencies in a diploid population. Mol Biol Evol 12:921-927.

Fortina P, Delgrosso K, Sakazume T, Santacroce R, Moutereau S, Su HJ, Graves D, McKenzie S, Surrey S (2000) Simple two-color array-based approach for mutation detection. Eur J Hum Genet 8:884-894.

Gabriel SB, Schaffner SF, Nguyen H, Moore JM, Roy J, Blumenstiel B, Higgins J, DeFelice M, Lochner A, Faggart M, Liu-Cordero SN, Rotimi C, Adeyemo A, Cooper R, Ward R, Lander ES, Daly MJ, Altshuler D (2002) The structure of haplotype blocks in the human genome. Science 296:2225-2229.

Hill WG, Robertson A (1968) Linkage disequilibrium in finite populations. Theor Appl Genet 38:226-231.

Hinds DA, Stuve LL, Nilsen GB, Halperin E, Eskin E, Ballinger DG, Frazer KA, Cox DR (2005) Whole-genome patterns of common DNA variation in three human populations. Science 307:1072-1079.

Jaakson K, Zernant J, Kulm M, Hutchinson A, Tonisson N, Glavac D, Ravnik-Glavac M, Hawlina M, Meltzer MR, Caruso RC, Testa F, Maugeri A, Hoyng CB, Gouras P, Simonelli F, Lewis RA, Lupski JR, Cremers FP, Allikmets R (2003) Genotyping microarray (gene chip) for the ABCR (ABCA4) gene. Hum Mutat 22:395-403.

Jorde LB (2000) Linkage disequilibrium and the search for complex disease genes. Genome Res 10:1435-1444.

Kaminski S, Ahman A, Rusc A, Wojcik E, Malewski T (2005) MilkProtChip-a microarray of SNPs in candidate genes associated with milk protein biosynthesis-development and validation. J Appl Genet 46:45-58.

Klitø NGF (2006) Implementing arrayed primer extension for specialised gene-phenotype association studies. $\mathrm{PhD}$ Thesis, 104 pages. Availability: Odense University Hospital library, http://www.sdu.dk/ videncenteret/english/index.php/.

Kurg A, Tonisson N, Georgiou I, Shumaker J, Tollett J, Metspalu A (2000) Arrayed primer extension: solid-phase four-color DNA resequencing and mutation detection technology. Genet Test 4:1-7.

Landi S, Gemignani F, Moreno V, Gioia-Patricola L, Chabrier A, Guino E, Navarro M, de Oca J, Capella G, Canzian F (2005) A comprehensive analysis of phase I and phase II metabolism gene polymorphisms and risk of colorectal cancer. Pharmacogenet Genomics 15:535-546.

Lewontin RC (1964) The interaction of selection and linkage. I. General considerations; heterotic models. Genetics 49:49-67.
Lovmar L, Fredriksson M, Liljedahl U, Sigurdsson S, Syvanen AC (2003) Quantitative evaluation by minisequencing and microarrays reveals accurate multiplexed SNP genotyping of whole genome amplified DNA. Nucleic Acids Res 31:e129.

Maron E, Nikopensius T, Koks S, Altmae S, Heinaste E, Vabrit K, Tammekivi V, Hallast P, Koido K, Kurg A, Metspalu A, Vasar E, Vasar V, Shlik J (2005) Association study of 90 candidate gene polymorphisms in panic disorder. Psychiatr Genet 15:17-24.

Pastinen T, Raitio M, Lindroos K, Tainola P, Peltonen L, Syvanen AC (2000) A system for specific, high-throughput genotyping by allelespecific primer extension on microarrays. Genome Res 10:10311042.

Schrijver I, Oitmaa E, Metspalu A, Gardner P (2005) Genotyping microarray for the detection of more than 200 CFTR mutations in ethnically diverse populations. J Mol Diagn 7:375-387.

Syvanen AC (2001) Accessing genetic variation: genotyping single nucleotide polymorphisms. Nature Rev Genet 2:930-942.

Syvanen AC (2005) Toward genome-wide SNP genotyping. Nature Genet 37:5-10.

Tebbutt SJ, He JQ, Burkett KM, Ruan J, Opushnyev IV, Tripp BW, Zeznik JA, Abara CO, Nelson CC, Walley KR (2004) Microarray genotyping resource to determine population stratification in genetic association studies of complex disease. Biotechniques 37:977-985.

The International HapMap Consortium (2005) A haplotype map of the human genome. Nature 437:1299-1320.

Thorisson GA, Smith AV, Krishnan L, Stein LD (2005) The International HapMap Project Web site. Genome Res 15:1592-1593.

Tonisson N, Kurg A, Kaasik K, Lõhmussaar E, Metspalu A (2000) Unravelling genetic data by arrayed primer extension. Clin Chem Lab Med 38:165-170.

Weiss KM, Clark AG (2002) Linkage disequilibrium and the mapping of complex human traits. Trends Genet 18:19-24.

Address reprint requests to: Dr. Niels G.F. Klitф

Department of Biochemistry, Pharmacology and Genetics

Human Microarray Centre

Odense University Hospital

Sdr. Boulevard 29

Dk-5000 Odense C, Denmark

E-mail: niels.klitoe@mail.dk 